Submitted to ApJ

Preprint typeset using $\mathrm{LAT}_{\mathrm{E}} \mathrm{X}$ style emulateapj v. 08/22/09

\title{
THE SIGNATURE OF GALACTIC TIDES IN LOCAL GROUP DWARF SPHEROIDALS
}

\author{
Jorge Peñarrubia ${ }^{1,2}$, Julio F. Navarro ${ }^{2,3}$, Alan W. McConnachie ${ }^{2,4}$ \& Nicolas F. Martin ${ }^{5}$ \\ Submitted to ApJ
}

\begin{abstract}
We use N-body simulations to explore the effects of tidal stripping on the structure of dwarf spheroidal galaxies (dSphs). Our models assume cosmologically motivated initial conditions where dSphs are modeled as King spheres, embedded in Navarro-Frenk-White dark halos and orbiting the Galactic potential on eccentric orbits. As expected, systems that orbit through dense regions of the Galaxy lose a significant fraction of stars after each pericentric passage. These episodes of mass loss, however, do not impose a clear tidal cutoff on the bound stellar core. Rather, once equilibrium has been re-established the outer mass profile approaches a power-law well described by a simple Plummer model. As noted in earlier work, tides also result in transient features in the outer density profile. As the system relaxes, an outward-moving "excess" of stars is found at radii where the local crossing time exceeds the time elapsed since pericenter. If the orbit of the dSph is known, these results provide a simple way to assess whether "breaks" and "bumps" in the outer profile of dSphs are actually tidal in origin. We apply this to the Sagittarius dwarf and, encouragingly, identify two features in the surface brightness profile that may be traced to its two last pericentric passages. Applied to Leo I, our results predict that any tidal break would occur at radii beyond those surveyed by current data, casting doubt on recent claims of the detection of tidal debris around this galaxy. For Carina, our model indicates that the tidal break should occur at a radius twice farther than observed. This suggests that the outer excess of stars in Carina is not tidal in origin unless its orbit is in error. A similar comment applies to Sculptor, whose pericenter appears too large for Galactic tides to be important but whose outer profile, like that of Draco, nonetheless follows closely a Plummer-law. Fornax and Leo II show no sign of a power-law outer profile, suggesting that they have not been significantly affected by tides. Published profiles for other Milky Way dSph companions do not extend sufficiently far to allow for conclusive assessment. Panoramic surveys that extend surface brightness profiles beyond $\sim 10$ core radii, together with improved constraints on the orbital parameters of dSphs, are needed in order to establish the true origin of the outer envelopes of stars surrounding dSphs.
\end{abstract}

Subject headings:

\section{INTRODUCTION}

The last decade has seen the emergence of a paradigm for the matter content and geometry of the Universe, where structures form as gravity amplifies primordial fluctuations in the dominant form of matter in the Universe: Cold Dark Matter (CDM). One virtue of the CDM paradigm is that, once its parameters are fixed by observations of large scale structures (Mpc and larger), it can be used to make precise predictions on small scales. Verifiable predictions on the scale of galaxies are particularly important, given the numerous observations that constrain the content and spatial distribution of dark matter in galaxies of various types.

The most discerning tests apply to faint galaxies. This is because the luminous (baryonic) component of galaxies is of lesser dynamical importance in these systems (the most dark-matter dominated galaxies known are dwarfs), and dynamical constraints may therefore be compared di-

\footnotetext{
Electronic address: jorpega@ast.cam.ac.uk

${ }^{1}$ Institute of Astronomy, University of Cambridge, Madingley Road, Cambridge CB3 0HA, UK

2 Department of Physics and Astronomy, University of Victoria, 3800 Finnerty Rd., Victoria, BC, V8P 5C2, Canada

${ }^{3}$ Fellow of the Canadian Institute for Advanced Research

${ }^{4}$ Herzberg Institute of Astrophysics, 5071 West Saanich Road, Victoria, BC V9E 2E7, Canada

${ }^{5}$ Max-Planck-Institut fuer Astronomie, Königstuhl 17, D-69117 Heidelberg, Germany
}

rectly with CDM predictions even when full understanding of the galaxy formation mechanism may be lacking. Further, the properties of galaxies and dark matter diverge on small scales: we expect many more small dark matter halos than there are known faint galaxies (White \& Rees 1978, Kauffmann et al 1993, Cole et al 1994). Understanding how one population maps onto the other would shed light on the nature of dark matter and on the main mechanisms responsible for galaxy formation.

This line of inquiry is potentially most fruitful when applied to the faintest galaxies known, since their properties are expected to be extraordinarily sensitive to the detailed ingredients of galaxy formation models and to the nature of dark matter. The dark matter content of dwarf spheroidal galaxies (dSphs) is thus an issue of crucial importance in galaxy formation studies.

Because they are extremely faint, dSphs can only be studied in the Local Group, where they appear to cluster tighly around M31 and the Milky Way. These systems are therefore thought to inhabit the small systems making up the abundant "substructure" seen in cosmological simulations of CDM halos (Klypin et al 1999, Moore et al 1999).

The sheer number of these substructure halos ("subhalos", for short) poses an interesting challenge: the most recent simulations indicate that the Milky Way halo may host thousands of subhalos massive enough, in princi- 
ple, to host faint galaxies, a number that far exceeds the known luminous Galactic satellites. One way of reconciling this discrepancy is to assume that luminous satellites inhabit only the (few) most massive subhalos (Stoehr et al 2002). Alternatively, the scarcity of luminous satellites may reflect the operation of a physical mechanism that "lights up" a small selection of subhalos with a wide range of masses depending, for example, on their collapse time, density, and/or environment (Bullock et al 2002, Benson et al 2002, Madau et al 2008). Strong constraints on the total mass and radial extent of the dark halos surrounding dSphs would help distinguish between these possibilities.

Stars are the only viable dynamical tracer in dSphs, so direct mass estimates rely on the kinematics and radial extent of their stellar component. This yields constraints on the total mass within the luminous radius of each dwarf but provides little information on the extent or mass of a surrounding halo (Peñarrubia, McConnachie \& Navarro 2008, hereafter Paper I). Probes of the outer halos of dSphs are, therefore, necessarily indirect, but crucial to elucidate the cosmological origin of these systems.

The response of satellites to the Galactic tidal field provides a promising way to make progress. Ever since dSphs were discovered (Hubble 1936, Holmberg 1950), Galactic tides have been thought to play a significant role in sculpting their structure. This assumption finds inspiration in the fact that the shape of the surface brightness profiles of dSphs resembles that of globular clusters, with a well-defined central region of nearly constant stellar density (a "core") and a sharp decline in the outer parts (e.g. Irwin \& Hatzidimitriou 1995). King (1962) profiles capture these features well, and have traditionally been the parametrization of choice for dSph profiles. The outer cutoff in the luminous profile has therefore often been identified with a "tidal radius" imposed by the Galactic potential, just like in King (1966) models of globular clusters.

This interpretation is, however, debatable, given that dSphs are substantially more massive than globulars and orbit mostly the outer Galactic halo on long period orbits. It is thus still unclear whether the outer limit in the stellar distribution of a dSph has true dynamical significance or whether it just reflects a sharp boundary in the region where star formation was able to proceed efficiently.

On the other hand, if Galactic tides are actively stripping stars from dSphs, it would be clear that these systems are unlikely to be surrounded at present by massive dark halos extending far beyond their luminous radius. This is because the outer parts of such halos would have been stripped long before the stars (see, e.g., Peñarrubia, Navarro \& McConnachie 2008, hereafter Paper II), implying that the total self-bound mass associated with a dwarf does not exceed greatly the dynamical mass estimated from its stellar size and kinematics. Dynamical masses have been measured for most dwarfs, and, intriguingly, seem almost independent of dSph luminosity (see, e.g., the review of Mateo 1998, Simon \& Geha 2007, Walker et al. 2007, Gilmore et al. 2007, Martin et al. 2007 and also our Paper I). This property seems to be shared by the ultra-faint dSph population recently discovered around the Milky Way (e.g Zucker et al. 2004, 2006a,b,c, Willman et al. 2005, Belokurov et al. 2006a, 2007, 2008; Irwin et al. 2007). Strigari et al (2008), for example, conclude that dSphs spanning almost 7 decades in luminosity share a common $\sim 10^{7} M_{\odot}$ mass scale within a $300 \mathrm{pc}$ radius. It would be interesting to see whether this result also holds for the dSph population recently discovered around M31 (Zucker et al. 2004, 2007; Martin et al. 2006; Majewski et al. 2007; Ibata et al. 2007; Irwin et al. 2008; McConnachie et al. 2008).

If this is truly the total mass associated with dSphs, then strong constraints on how these galaxies formed would follow. Cosmological simulations indicate that one may expect roughly one thousand $\sim 10^{7} M_{\odot}$ CDM subhalos around the Milky Way (Diemand et al 2008, Springel et al 2008), challenging models to explain what led only a tiny fraction of such systems to host luminous dwarfs. Confirming whether dSphs are actually losing stars to Galactic tides, and therefore inhabit low-mass subhalos, would thus provide a really important clue to the origin of these systems.

We examine these issues here with the aid of $\mathrm{N}$ body simulations of cosmologically-motivated dSph models, where the stellar component is represented by King (1966) spheres embedded within Navarro, Frenk \& White (1996, 1997, hereafter NFW) dark matter halos. Our main goal is to identify unambiguous signatures of the effect of Galactic tides on the stellar structure of a dwarf spheroidal. In particular, we examine the effect of tidal mass loss on the surface brightness profile, as well as the significance of transient "bumps" and "breaks" in the outer profile caused by tides. Such features have been reported in the literature for a number of dwarfs, but it is still unclear how or whether they relate to the effect of Galactic tides.

The plan for this paper is as follows. In Sec. 2 we introduce our numerical models, and outline the main results in Sec. 3. We apply these results in Sec. 4 to individual Milky Way dSph satellites. Sec. 5 summarizes our main findings.

\section{NUMERICAL SIMULATIONS}

Our numerical setup has been presented in detail in Paper II and we refer the interested reader to that contribution for details. For completeness, and in order to introduce useful notation, we describe here the main aspects of the modeling procedure.

\subsection{The host galaxy}

We model the host galaxy potential with an NFW profile, given by

$$
\Phi_{\mathrm{NFW}}(r)=-V_{\max }^{2} \frac{r_{\max }}{r} \ln \left(1+r / r_{s}\right),
$$

where $V_{\max }$ and $r_{\max }$ identify the peak of the circular velocity profile. The radial scale $r_{\max }$ is $2.16 r_{s}$, the usual scale radius of the NFW profile. The host potential is assumed fixed during the evolution of the dwarf. Since our main goal is to identify the signatures of Galactic tidal stripping in dwarfs, for simplicity we shall neglect in this contribution the effects introduced by subhalo-subhalo interactions $^{6}$. Our models are purely gravitational and

\footnotetext{
6 We refer interested readers to Peñarrubia \& Benson 2005; Mastropietro et al. 2005; González-García et al. 2005; Aguerri \&
} 
therefore scale-free. When convenient for interpretive purposes, we scale our numerical units to physical parameters similar to those estimated for the Milky Way by Klypin, Zhao \& Somerville (2002): $V_{\max }=188 \mathrm{~km} / \mathrm{s}$ and $r_{\max }=18 \mathrm{kpc}$.

\subsection{Dwarf galaxy model}

Our dSph models assume that the stellar component follows a King (1966) sphere embedded within a CDM halo. The dark halo is modeled as an N-body realization of a spherical NFW profile and is constructed by generating a 5 million-particle equilibrium realization using a code kindly made available by S.Kazantzidis (Kazantzidis et al. 2004, 2006). Using the same physical scaling adopted for the host galaxy (§ 2.1) we assume for the dwarf halo that $r_{\max }^{d}=4.4 \mathrm{kpc}$ and $V_{\max }^{d}=28.6$ $\mathrm{km} / \mathrm{s}$. Hereafter we use the superscripts $d$ and $h$ to identify parameters corresponding to the dwarf and the host, respectively.

Motivated by the large mass-to-light ratio $(M / L)$ exhibited by the Milky Way dSphs $(20 \lesssim M / L \lesssim 1000$; Mateo 1998; Simon \& Giha 2007) we assume that stars contribute negligibly to the potential, and may therefore be followed by assigning an energy-dependent $M / L$ to each dark matter particle in the equilibrium halo (see Paper II for details). The outcome is a subset of particles that follows a King model in equilibrium within the dark halo. The surface density profile of the initial stellar component can be written as

$$
\Sigma(R)=k\left\{\left[1+\left(R / R_{k}\right)^{2}\right]^{-1 / 2}-\left[1+\left(R_{t} / R_{k}\right)^{2}\right]^{-1 / 2}\right\}^{2}(2)
$$

where $R$ is the projected radius and $k$ an arbitrary constant. We consider models with values for the King "concentration" $c_{K} \equiv R_{t} / R_{k}=5$ and 10 , but note that none of our main conclusions depends on this choice. For an easier comparison with observations, we also use the core radius $R_{c}$, which is estimated independently of any fitting procedure as the radius where the surface density drops to one half of its central value. Note that for a King model $R_{c} \rightarrow R_{k}$ as $c_{k} \rightarrow \infty$. For concentrations $c_{k}=5$ and $10, R_{c} / R_{k}=0.84$ and 0.92 , respectively.

The main parameter of the model is the degree of spatial segregation between stars and dark matter in the dwarf, which we may express as the ratio between the stars' King radius, $R_{k}$, and $r_{\text {max }}^{d}$, the radius where the halo circular velocity peaks. The segregation between stars and dark matter is constrained by the shape of the stellar velocity dispersion profiles. As discussed in Paper I, only systems where stars are deeply embedded within the dark halo have projected velocity dispersion profiles, $\sigma_{p}(R)$, that, in agreement with observations, remain nearly flat almost out to the "tidal" radius. For the eight Milky Way dSphs examined in Paper I, we show that spatial segregations in a broad range, $0.02 \leq R_{k} / r_{\max }^{d} \leq 0.14$, satisfy this constraint.

From a numerical point of view, the more deeply segregated the stellar component is, the fewer particles are available to trace it, and therefore large numbers of particles are required to resolve the innermost regions of

González-García 2009 for interesting discussions on the role played by tidal harassment in the dynamical evolution of satellite galaxies halos where the stars are thought to reside. Using a total number of $5 \times 10^{6}$ dark matter particles for the NFW profile we obtain stellar components traced by $1.95 \times 10^{4}$, $6.86 \times 10^{4}$, and $1.88 \times 10^{5}$ particles, for $R_{k} / r_{\max }^{d}=0.02$, 0.05 , and 0.10 , respectively.

\subsection{The Orbits}

The dwarf galaxy models are placed on highly eccentric orbits of fixed apocenter, $r_{\text {apo }}=10 r_{\max }=180 \mathrm{kpc}$. We ran several simulations in order to explore the result of varying the pericentric distance. As expected, the smaller the pericenter of the orbit the stronger the tidal effects. This may be quantified by the theoretical "tidal radius" $\left(r_{\text {tid }}\right)$, defined by (Paper II)

$$
\left\langle\rho^{\mathrm{d}}\right\rangle\left(r_{\text {tid }}\right)=3\left\langle\rho^{\mathrm{h}}\right\rangle\left(r_{\text {peri }}\right),
$$

where $\langle\rho\rangle(r)$ denotes the mean enclosed density within $r$. Only when $r_{\text {tid }}$ is comparable to the size of the stellar component will the dwarf experience a sizeable change in the structure of its stellar component. Since we are interested in systems that do lose stars to tides after the first pericentric encounter, we concentrate here on orbits with rather small pericenters; i.e., $r_{\text {peri }}: r_{\text {apo }}=1: 50,1: 100$ and 1:200, which lead to substantial mass loss. Table 1 summarizes the main parameters of each run. The table lists the orbital parameters of each orbit, as well as the fraction of dark mass contained initially within $r_{\text {tid }}$. It also lists the fraction of stars within $r_{\text {tid }}$ for our choice of segregation parameter $R_{k} / r_{\max }^{d}=0.1$, and two possible values of the King concentration, $c_{K}=5$ and 10 . These models are integrated only until their first orbital apocenter. When convenient, we also use some of the dwarf galaxy models introduced in Paper II, which follow the evolution of dwarf galaxies for several orbital periods.

\subsection{The $N$-body code}

We use SUPERBOX, a highly efficient particle-mesh code (see Fellhauer et al. 2001 for details) to track the evolution of our dSph N-body models in the host potential. SUPERBOX uses three nested grid zones with different size centered on the highest-density particle cell of the dwarf. This center is updated every time-step, so that all grids follow the satelly galaxy along its orbit.

Our numerical setup is outlined in detail in Paper II. For completeness we summarize here the main numerical parameters. Each of the three grids has $128^{3}$ cells. (i) The inner grid resolves the inner region of the dwarf and has a spacing of $d x=r_{s}^{d} / 126 \simeq 8 \times 10^{-3} r_{s}^{d}$. (ii) The middle grid covers the whole dwarf with a spacing that is $d x=r_{\text {vir }}^{d} / 126$. (iii) lastly, the outermost grid extends out to $50 r_{\text {vir }}^{d}$ and is meant to follow particles that are stripped from the dwarf and orbit within the host galaxy. SUPERBOX uses a leap-frog scheme with a constant time-step to integrate the equations of motion, which is selected according to the criterion of Power et al. (2003). Applied to our dwarf galaxy models, this yields $\Delta t=4.6 \mathrm{Myr}$. In Paper II we include numerical checks that show that the above selection of parameters leads to N-body dSph models that are in dynamical equilibrium and do not evolve away from the original configuration in isolation.

To simplify the analysis of the N-body experiments, we define mass, size, velocity and time N-body units as 


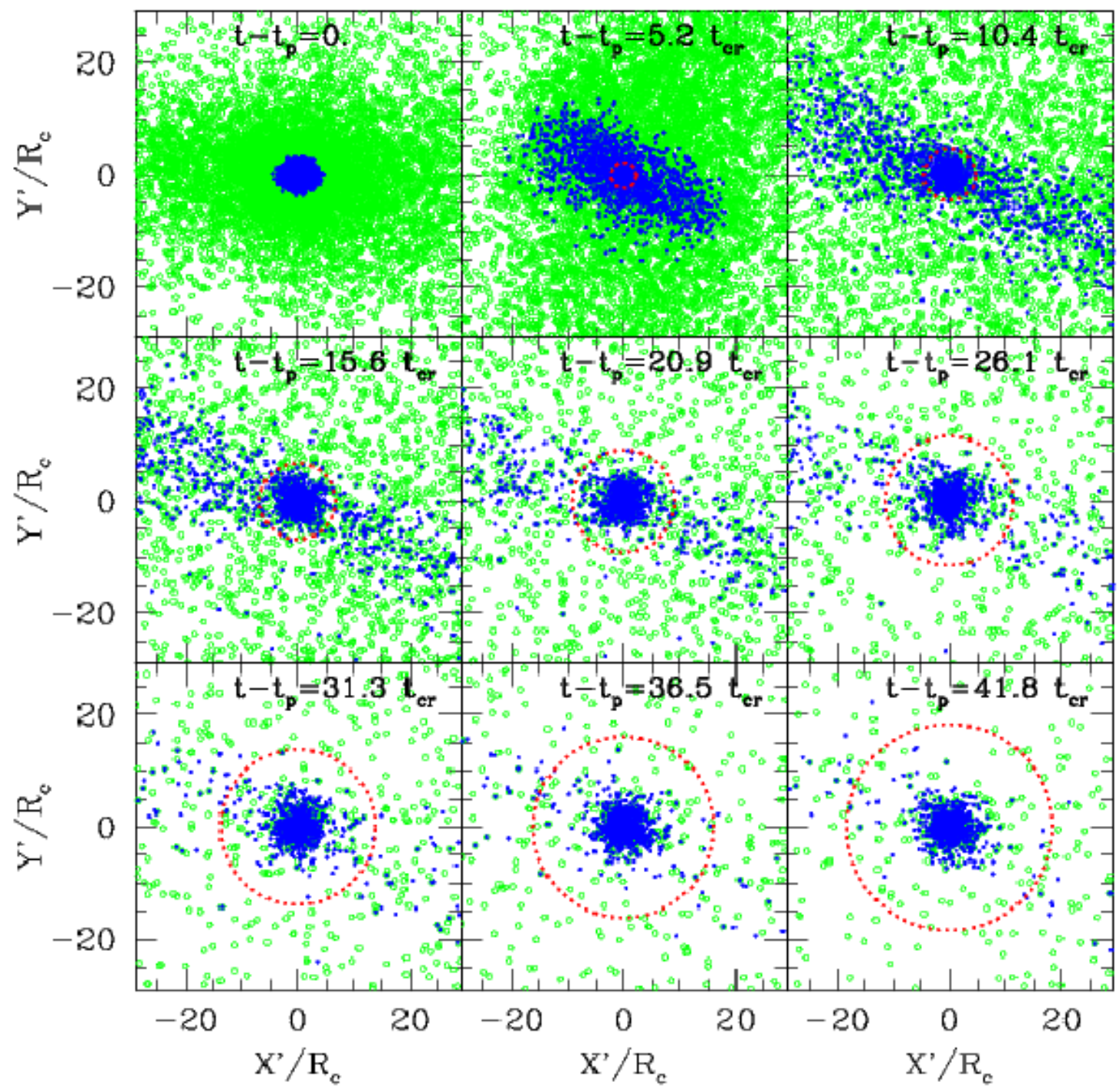

FIG. 1. - Time sequence illustrating the evolution of a dSph on a highly-eccentric $\left(r_{\text {peri }}: r_{\text {apo }}=1: 200\right)$ orbit. Stars and dark matter particles are shown in the plane of the orbit and are plotted with filled and open circles, respectively, assuming an initial stellar segregation of $R_{k} / r_{\max }^{d}=0.1$. Each panel corresponds to a different time, is centered on the bound stellar core, and is labeled by the time elapsed since pericenter, $t-t_{p}$, given in units of the crossing time $t_{\mathrm{cr}}=R_{c} / \sigma_{0}$ of the dwarf. Dotted circles indicate the position of the "break" radius in the density profile discussed in the text.

$[M]=5.6 \times 10^{1} 0 M_{\odot},[R]=3.5 \mathrm{kpc},[V]=262 \mathrm{~km} / \mathrm{s}$ and $[T]=0.013$ Gyr. In these units, the gravitational constant is $G=1$.

\section{RESULTS}

\subsection{Tidal effects on eccentric orbits}

Fig. 11illustrates the evolution of one of our dwarf models after first pericentric passage on a highly eccentric orbit. Dark halo particles and "stars" are shown with open and filled circles, respectively, assuming a star-todark matter segregation parameter, $R_{k} / r_{\max }^{d}=0.1$ and $c_{K}=5$ for the King model representing the stars. The case shown corresponds to a rather extreme orbit, where the dwarf is stripped of $\sim 98 \%$ of its initial dark mass and $\sim 60 \%$ of its stars during the first pericentric passage. Particles are shown projected on the plane of the orbit, at various times following pericenter. Each panel is labelled by the time elapsed since pericenter, $t-t_{p}$, expressed in units of the crossing time of the stars at the core radius, as measured by $t_{\mathrm{cr}} \equiv R_{c} / \sigma_{0}$, where $\sigma_{0}$ is the central line-of-sight stellar velocity dispersion ${ }^{7}$. To let the models resettle into dynamical equilibrium, we measure these quantities at orbital apocenter.

The panels in Figure 1 show that, for such eccentric orbits, the effect of tides can be well approximated by an impulsive perturbation acting at pericenter. Indeed, before $t=t_{p}$ the stellar system shows almost no sign of being perturbed. A short time after pericenter, the stars and dark matter that have gained energy during the interaction start leaving the dwarf through two prominent

7 To minimize numerical noise, we calculate the central velocity dispersion at $R_{c} / 2$. Since our dwarf models exhibit a flat velocity dispersion profile $(2.2$ this particular choice of radius has a negligible impact on the value of $\sigma_{0}$ 


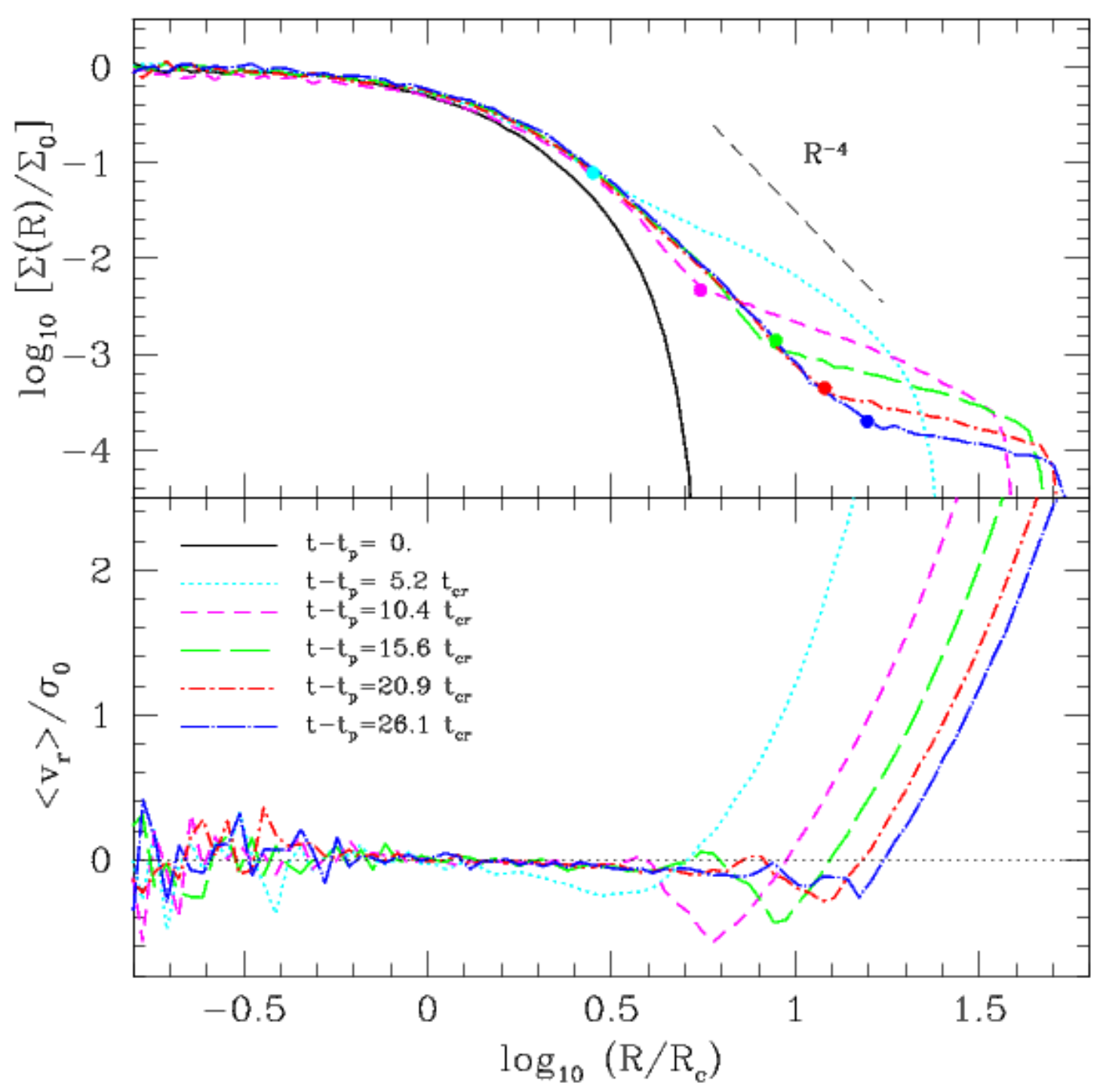

FIG. 2.- Upper panel: Projected stellar density profilesof the models shown in Fig. 1. Note how an initial King model model (solid line) evolves gradually towards an $R^{-4}$ power-law profile (dashed segment) because of tidal stripping. Filled circles indicate the "break" radius where the profile deviates significantly from the equilibrium profile the dwarf approaches at late times. Lower panel: Average radial velocity of stars (measured in 3D spherical shells) in the rest frame of the bound stellar core, as a function of radius. This panel shows that the excess of stars at large radii is due to particles moving away as they escape or settle gradually into their new, more loosely bound orbits.

"tidal tails".

Once enough time has elapsed to allow unbound material to leave the galaxy, the prominence of the tidal tails wanes and the remaining bound core of stars resettles into equilibrium. Few obvious "tidal features" remain then. At $t-t_{p}=41.8 t_{\mathrm{cr}}$ (bottom right panel in Figure 1) it would be difficult to guess that the dwarf has lost $60 \%$ of its original stars (see Paper II). As anticipated in Sec. 1, note that almost no dark matter is left surrounding the bound stellar core.

The process recurs after every pericentric passage. We refer the interested reader to Paper II for details regarding how the structural parameters of the dwarf (core radii, velocity dispersion, etc) evolve after each mass loss episode. We concentrate below on the tidally-driven evolution of the surface brightness profile of the stellar com- ponent.

\subsection{The equilibrium profile of a tidally-stripped $d S p h$}

The upper panel of Fig. 2 shows the stellar surface density profile (projected onto the orbital plane) at the various snapshots shown in Fig. 1. Each curve corresponds to a different time, after normalizing to the current values of the central density and of the core radius. The solid curve is the original undisturbed King profile.

This panel illustrates a few interesting points. The most obvious is that the stellar profile changes substantially as a result of the interaction. The outer regions of the profile become distended, although the shape in the inner regions is much less affected. As discussed in Paper II, force-fitting a King-model to the post-perturbation profile would result in much larger values of the King concentration, $c_{K}=R_{t} / R_{k}$. It is clear, however, that 

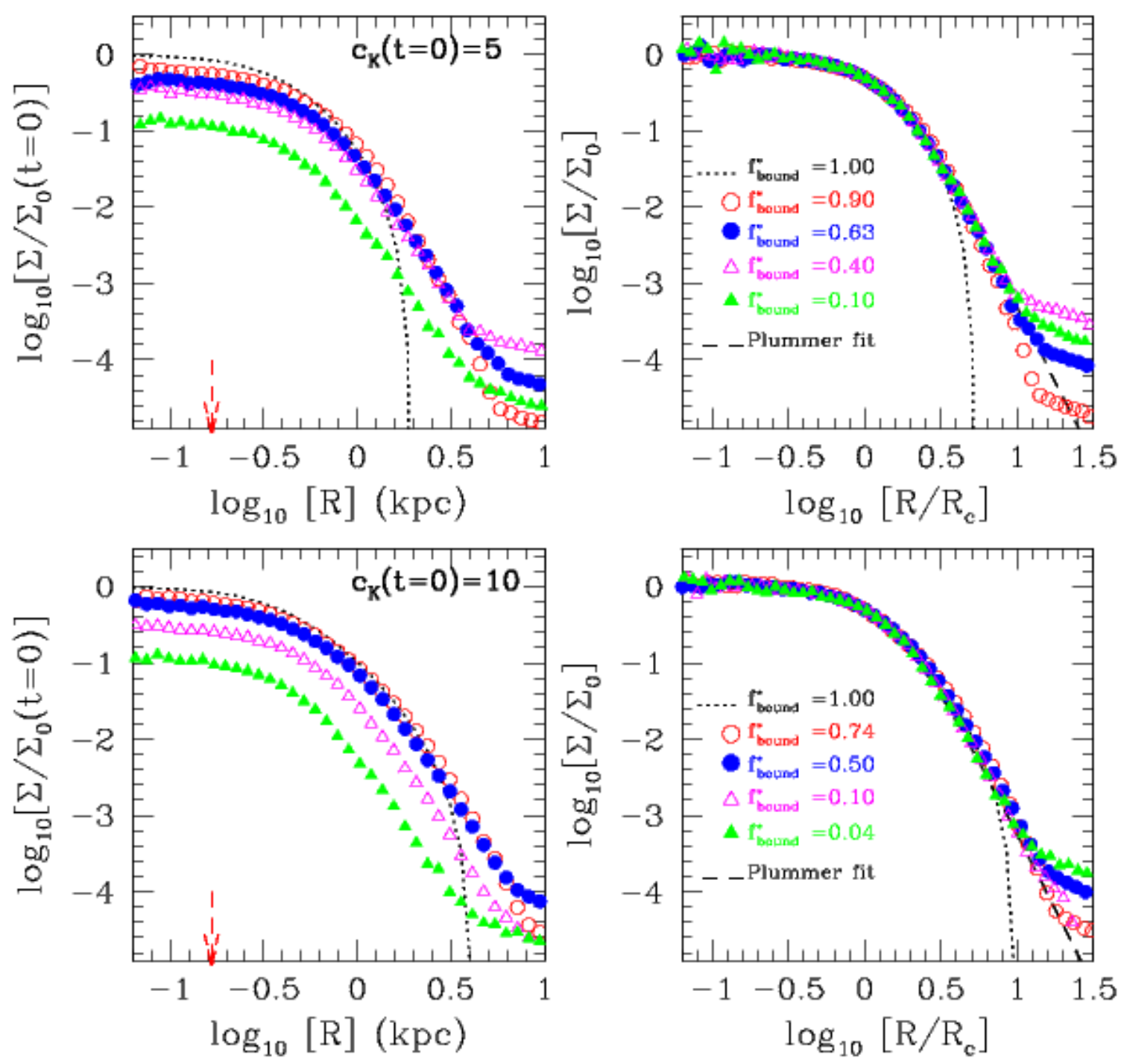

FIG. 3. - Left panels: Stellar surface density profiles of dSphs that have lost various fractions of their total mass to tides (indicated by the remaining bound stellar fraction, $f_{\text {bound }}^{*}$. The initial King model is shown with black dotted lines. Profiles are normalized to the initial central surface density $\Sigma_{0}(t=0)$. Upper and lower panels show models with different initial King concentrations $c_{K}$. Right panels: Same profiles as in left, but normalized to the current values of the central surface density and of the core radius. The latter is defined as the radius where $\Sigma$ drops to one-half of its central value. These panels show that (i) the initial truncation of the light profile quickly disappears as a result of tides, and that (ii) the bound stellar remnants evolve towards a spatial distribution that follows a $\Sigma \sim R^{-4}$ profile at large radii.

as a result of stellar stripping the outer profile loses the well-defined cutoff characteristic of a King sphere, and approaches instead a power-law at late times (see also Oh et al. 1995). This is not unexpected; the tidal impulse does not only remove some stars by endowing them with speeds exceeding the local escape velocity, but also shifts a number of stars to very loosely bound orbits, in effect populating phase-space all the way to zero energy, $E \sim 0$. A simple calculation shows that, under these circumstances, a power-law outer density profile may be expected once the dwarf re-equilibrates (White 1987; Jaffe 1987). The dashed segment shows that $\Sigma \propto R^{-4}$ describes the outer equilibrium envelope well.

All dSph models that have lost an appreciable fraction of stars to tides seem to develop an outer power-law profile. This is shown in Fig. 3, where we show the projected stellar density profile of several dwarfs after being perturbed by tides. The profiles are computed at apocenter, after the bound stellar core has relaxed to equilibrium, in order to minimize the presence of transients in the outermost region. (We discuss these transients below.) Profiles on the left panels of Fig. 3 are unscaled, and show how the system changes as mass is lost. (The unperturbed system is shown by a dotted line.) As discussed in Paper II, mass loss leads to a drop in the central surface density but leaves the core radius of the object almost unchanged. The top and bottom panels of this figure correspond to dSph models with initial King concentrations $c_{K}=5$ and 10, respectively. Each curve is labeled by $f_{\text {bound }}^{*}$, the fraction of their initial stellar mass 
that remains attached to the bound core.

As anticipated above, the outer regions of the profile become more distended, and approach a power-law. Scaling each profile to its central density and to its core radius (right-hand panels in Fig. 3 ) shows that a single law reproduces the equilibrium profiles of all models (provided they have experienced at least mild mass loss). A simple parametrization is provided by the projected Plummer (1911) law, which we write as

$$
\Sigma_{P}(R)=\frac{\Sigma_{0}}{\left[1+\left(R / R_{p}\right)^{2}\right]^{2}} .
$$

This conclusion seems independent of the initial concentration of the King sphere and of the actual orbit. Indeed, the profiles shown in Fig. 3 correspond to different orbits and are shown at different times, having been selected only to span a wide range in mass loss.

We conclude that the density profiles of relaxed, tidally-stripped dwarf spheroidals are well approximated by a Plummer law. This seems to be a necessary (but not sufficient) condition for identifying systems in eccentric orbits that have lost a significant amount of mass to stripping. Interestingly, this result implies that those dSphs where the outer cutoff in the luminosity profile is sharpest are likely those that have been least affected by tidal effects.

\subsection{Tidally-induced transients in the stellar profile}

Another obvious feature in the top panel of Fig. 2 is the presence of a transient "excess" in the outer density profile over the power-law equilibrium profile to which the system relaxes. The location where the excess becomes noticeable may be seen as a "break" in the outer profile that appears to march outward with time. The existence of breaks in the light distribution has been often interpreted as a sign of tidal stripping (Johnston et al. 2002; Mayer et al. 2002; although see McConnachie et al. 2007 for an alternative scenario) and may be traced to stars that have gained energy at pericenter and are still moving away as they settle into their new, less bound orbits within the dwarf (or escape altogether). This may be seen in the bottom panel of Fig. 2, which shows the mean values of the radial velocity component in the dwarf frame as a function of projected radius for the various snapshots shown in the top panel. Clearly, the location of the "break" in the profile corresponds roughly to where the average motion of stars transitions from equilibrium $\left(\left\langle v_{r}\right\rangle \approx 0\right)$ to becoming dominated by outward motion $\left(\left\langle v_{r}\right\rangle>0\right)$.

As discussed in detail by Aguilar \& White (1986, see also Navarro 1990), the position of the "break" correlates with the time elapsed since pericenter. This provides a "clock" that may be used to verify whether "breaks" in observed dSph profiles are actually tidal in origin. This is shown in Fig. 4, where we plot the position of the break, $R_{b}$, (which is easily measured from the density profile; see filled circles in the top panel of Fig 2 versus the time elapsed since pericenter, $t-t_{p}$. The plot shows the results of several dSph models on different orbits, at various stages of disruption. The top and bottom panels of Fig. 4 show the same data; unscaled in the top panel and scaled to the core radius and core crossing time in the bottom panel. The position of the break radius and the time elapsed since pericenter are tightly correlated, so that a simple linear fit,

$$
R_{b}=C \sigma_{0}\left(t-t_{p}\right),
$$

with $C=0.55 \pm 0.03$ approximates well the data in Fig. 4 (see dashed line). Although the fit is generally good, it gets better at late times. Note that at times shortly past pericenter, when the break is close to the core, eq. 5 tends to underestimate the true radius of the break; by up to $\sim 40 \%$ for $R_{b} \lesssim 4 R_{c}$

Nevertheless, it is clear that, regardless of the details of the disruption process, the local crossing time at the location of the break, $R_{b} / \sigma_{0}$, is an extremely good indicator of the time elapsed since pericenter. If the orbit and the velocity dispersion of a dSph are known, then eq. 5 offers a simple way of testing whether a "break" seen in the outer profile is tidal in origin.

\section{APPLICATION TO LOCAL GROUP DWARFS}

The results presented above indicate that two conditions must be satisfied for a "break" in the outer profile to be considered tidal in origin. (i) The dwarf must be susceptible to tides; i.e., the "tidal radius", $r_{\text {tid }}$ (eq.3), at perigalacticon should be comparable or smaller than the luminous radius of the dwarf. (ii) The break must occur at a radius where the local crossing time is compatible with the time elapsed since perigalacticon.

The absence of a "break" does not, of course, necessarily imply that a dwarf has not lost stars to tides. Because the "break" radius moves quickly outward, it would be easy to miss as soon as it drifts into a region where the surface density of stars is low. For the best studied dwarfs, profiles can be traced robustly out to $\sim 10$ core radii, where the surface density is typically about $10^{-4}$ times lower than at the center. The tidal "break" in the profiles would therefore only be detectable for roughly 20 core crossing times after pericenter; i.e., for $t-t_{p} \sim 20 R_{c} / \sigma_{0}$. This corresponds to $3 \mathrm{Gyr}$ for a system as bright as Sagittarius, but to only $300 \mathrm{Myr}$ for a smaller system like Sculptor. Detecting unambiguous "tidal breaks" thus requires either catching a dwarf soon after pericentric passage or else deep panoramic surface photometry to measure robustly the profile at very large radii.

In order to keep the analysis simple, we have used throughout this analysis profiles onto the plane of the satellite's orbit. This facilitates the separation between material bound and unbound to the satellite and sharpens the features in the profile by minimizing the chance projection of foreground or background unrelated stars and the associated effects (Read et al. 2006; Muñoz et al. 2008). Such complication, of course, would need to be considered in detailed studies of individual objects (e.g. Klimentowski et al. 2007; Łokas et al. 2008), but is beyond the scope of this paper.

In the case where a break is absent, a tidally-affected dwarf could still be identified through the presence of a well-defined Plummer outer law profile. Of course, this is not a sufficient condition (why couldn't a dSph be born with a Plummer profile?), but it may help to pinpoint those systems for which further studies might be particularly fruitful. We caution, however, that distinguishing a Plummer profile from a King model where the tidal radius is much larger than the core radius might not be 

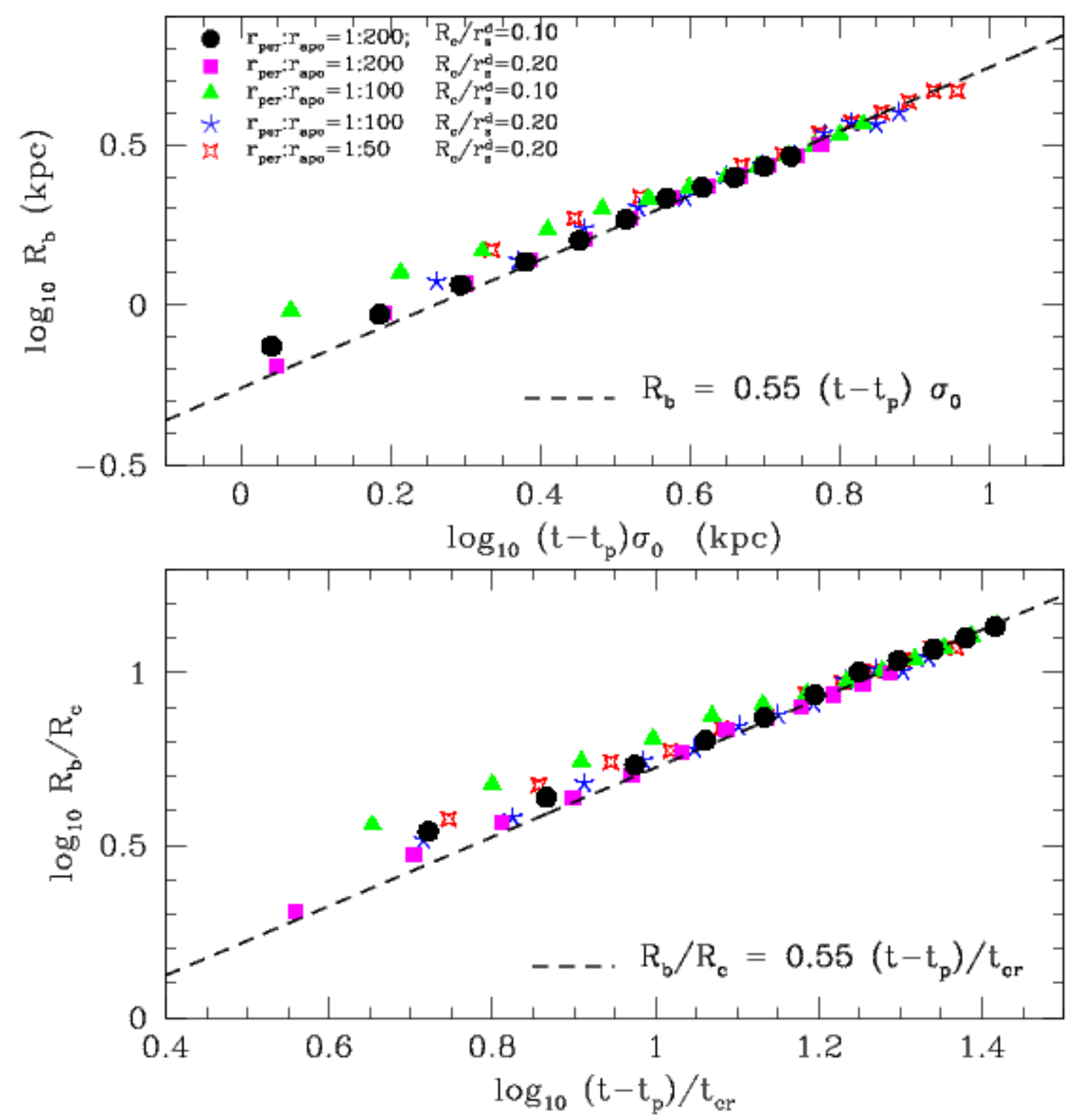

FIG. 4.- Position of the "break radius", $R_{b}$, measured from the sudden upturn in the density profiles (see, e.g., the top panel of Fig. 2, as a function of the time elapsed since pericenter times the central velocity dispersion $\left(t-t_{p}\right) \sigma_{0}$ (upper panel). The dotted line shows the result of a simple linear fit given by eq. 5 For easier comparison with observations, we also show in the bottom panel the same data points normalized by the dwarf core radius, $R_{c}$, and by the core crossing time, $t_{c r} \equiv R_{c} / \sigma_{0}$. This result provides a clock that may be used to assess whether perturbations in the outer profiles of dSphs are actually tidal in origin.

easy. The bottom-right panel of Fig. 3 illustrates this. Distinguishing a $c_{K}=10 \mathrm{King}$ model (dotted line) from a Plummer (dashed curve) would require examining the profile at surface densities below $10^{-4}$ times the central value. Very few systems have data of such quality. On a more optimistic note, according to our analysis, dSphs with sharply truncated outer light profiles are unlikely to have been affected by tides.

With these caveats in mind, we now apply these lessons to the case of Milky Way dSphs. For dwarfs with available proper motions (see Table 2) we compute their orbits assuming a Klypin et al. (2002) model for the Milky Way potential. In our orbit estimates we neglect in all cases the effect of dynamical friction, since the mass of these galaxies is very low and we are only interested in the (short) time elapsed since last perigalacticon. The tidal radius $r_{\text {tid }}$ of each $\mathrm{dSph}$ is calculated using the mass profiles derived in Paper I. We begin by validating our conclusions on the Sagittarius dwarf and discuss next a few other galaxies with published orbits. We conclude with a discussion of how these results may apply to the population of ultra-faint dwarfs recently discovered in the Milky Way halo.

\subsection{The case of Sagittarius}

The Sagittarius dwarf (Sgr, for short) offers an ideal testbed for the ideas presented in the previous section. Sgr is not only the brightest dSph and one of the closest, but also the one system where evidence for ongoing tidal stripping is definitive and uncontroversial owing to the detection of associated tidal streams (see, e.g., Belokurov et al. 2006b and references therein). Its surface density profile has been traced to very large radii using 2MASS data (Majewski et al. 2003) and, because of its proximity 


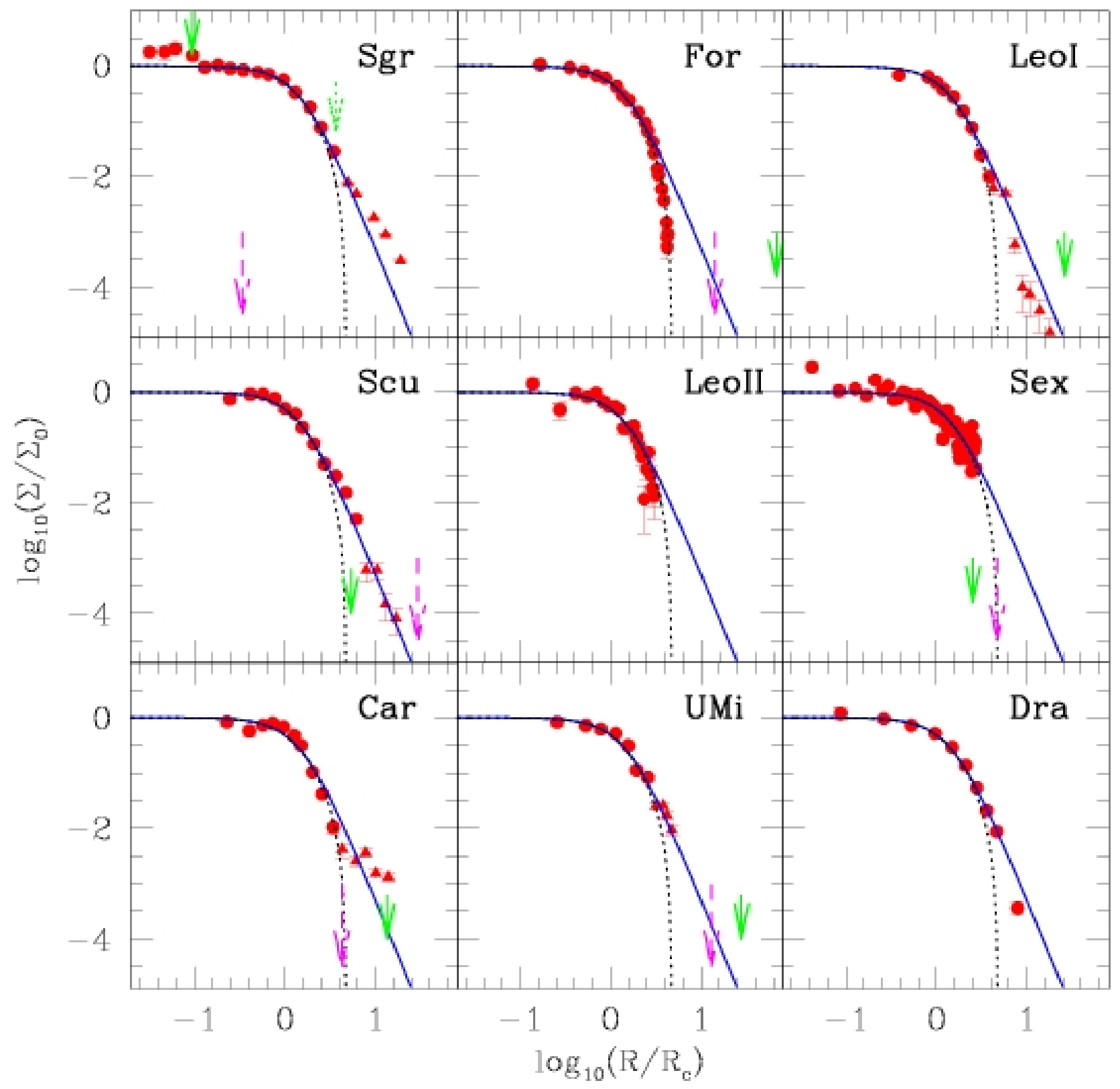

FIG. 5. - Surface brightness profiles of Sagittarius (from Majewski et al. 2003), Fornax (Battaglia et al. 2006), Leo I (Sohn et al. 2007), Sculptor (Westfall et al. 2006), Leo II (Coleman et al. 2007), Sextans (Irwin \& Hatzidimitriou 1995), Carina (Muñoz et al. 2006), UMi (Palma et al. 2003) and Draco (Martin et al. 2008). All profiles have been scaled to their central surface density and to the core radius, defined as the radius where $\Sigma$ drops to one half of its central value. Solid circles and triangles distinguish, respectively, between material within and beyond the "break" radius reported in the literature $\left(R_{b, \text { obs }}\right)$. In each panel, the solid line shows a Plummer law and the dotted line a $c_{K}=4 \mathrm{King}$ model. For systems with available proper motions, solid arrows indicate the position of the break radius predicted from eq. (5) (see also Table 2). For Sagittarius, we also plot a dotted arrow to show the estimated position of $\left(R_{b, \text { pred }}\right)$ associated to the penultimate pericentric passage. Dashed arrows denote the position of the tidal radius $r_{\text {tid }}$ at orbital perigalacticon. Only dwarfs where $r_{\text {tid }}$ is comparable or smaller than the luminous radius are expected to be susceptible to tidal stripping. 
( $\simeq 25 \mathrm{kpc}$ ), its orbit is also fairly well known (Dinescu et al. 2005). Sgr moves on an orbit that takes it from $\sim 17$ $\mathrm{kpc}$ from the Galactic center at perigalacticon to $\sim 55$ kpc at apogalacticon. It last went through perigalacticon $\sim 26 \mathrm{Myr}$ ago and its radial period is $\sim 1.0$ Gyr. The velocity dispersion of Sgr's bound core is $\sigma_{0} \sim 9.6 \pm 1$ $\mathrm{km} / \mathrm{s}$ (Bellazzini et al. 2008)

According to eq. 5, this implies that Sagittarius should exhibit a tidal "break" at $R_{b}=0.14 \mathrm{kpc}$ from the center. Note that this is well inside Sgr's core radius, which is estimated to be $\sim 1.5 \mathrm{kpc}$. Interestingly, Majewski et al. (2003) report the existence a "bump" in the stellar profile at about the same radius (marked by the leftmost arrow in the top-left panel of Fig. 5). Although the origin of the bump is unclear, our models suggest that it might be tidal in origin. If true, many stars around the "bump" should be in the process of leaving the dwarf, an assertion that could in principle be corroborated with velocity data.

The last pericentric passage is thus too recent to have altered the profile of the Sgr dwarf at large radii. However, the crossing time of Sgr is considerably long $(\simeq 150$ Myr), which suggests that the presence of the "break" associated to the penultimate perigalacticon passage may still be detectable in the outskirts of this galaxy. According to our orbit estimate, the penultimate perigalacticon passage occurred 1 Gyr ago, so that eq. 5 predicts a break radius at $R_{b} \sim 5.4 \pm 2.0 \mathrm{kpc}$ (dotted arrow in the top left panel of Fig. 5). This is slightly smaller than the $R_{b} \sim 7.3 \mathrm{kpc} \simeq 5 R_{c}$ break radius claimed by Majewski et al. (2003) (in this and other panels of Fig. 5 we use filled circles and triangles to report data within/outside the radius of the break reported in the literature cited in the caption). However, the difference is small, considering the ambiguities in pinning down the exact location of the break observationally, as well as the fact that eq. 5 tends to underestimate the location of the break when it occurs as close to the core as in the case of Sagittarius.

Finally, as a sanity check, the dashed arrow in the Sgr panel of Fig. 5 shows the location of the "tidal radius" computed using eq. 3 at perigalacticon. This is only of order 400 pc, well inside Sgr's stellar core radius, implying that Sgr is doomed to lose most of its stellar mass in subsequent orbits.

\subsection{Other Milky Way dSphs}

We analyze here the other five dSphs (aside from Sagittarius) where a "break" in the outer profile has been reported in the literature. Information on these dwarfs (Leo I, Sculptor, Carina, Ursa Minor, and Fornax) is listed in Table 2. Of the six, only Leo I has no available measurement of its proper motion, although its large Galactocentric distance (255 kpc, Caputo et al. 1999; Held et al. 2001; Bellazzini et al. 2004) together with its extreme heliocentric radial velocity $\left(v_{\mathrm{rad}, \mathrm{h}} \simeq 283 \mathrm{~km} / \mathrm{s}\right.$, Koch et al. 2007; Mateo 1998, 2008), are suggestive of a highly radial orbit. We can therefore safely neglect the tangential velocity component when estimating the time elapsed since the last pericentric passage.

The surface density profiles of the six dwarfs with claimed "breaks" are shown in Fig. 5. together with those of three other well-studied dSphs (Draco, Leo II and Sextans). Each profile has been scaled to its individual central surface density and core radius, for ease of comparison. Solid and dotted lines show, in each panel, a Plummer and a King $\left(c_{K}=4\right)$ model, respectively. For systems with full orbital information, we also show the position of the break radius as predicted from eq. (5) (solid arrows) and the tidal radius, $r_{\text {tid }}$ (eq.3), calculated at perigalacticon (dashed arrows).

\subsubsection{Fornax}

The Fornax dwarf provides probably the clearest case of a dwarf whose stellar component has not been disturbed by Galactic tides. Not only is the tidal radius clearly well outside the luminous radius, but also there is no evidence for distensions in the outer profile that may be ascribed to the past effect of tides. A $c_{k}=4 \mathrm{King}$ model fits the surface density profile of Fornax extremely well, down to the last measured point.

\subsubsection{Leo $I$}

The case of Leo I is less clear-cut. Sohn et al. (2007) report a "break" in the outer regions, which we highlight with triangles in the corresponding panel of Fig. 5. This figure shows that there is indeed an excess relative to a King model fit to the inner profile, but not relative to a Plummer law. Application of eq. 5 suggests that a tidal break should be located well beyond $10 R_{c}$, and thus outside the region surveyed around this dwarf. This implies that the outer profile of Leo I should be in dynamical equilibrium, and that no transients of tidal origin should be detectable in the data presently available.

This does not exclude the possibility that Leo I might have been tidally disturbed in the past. Indeed, its outer profile resembles closely the Plummer profile which, according to our results, tidally disturbed systems should evolve to. Interestingly, Mateo et al. (2008) find an increase of the orbital anisotropy for the stellar population located beyond the reported "break" radius. Further data that extend out into the region where local crossing times are comparable to the time elapsed since pericenter are needed to determine whether this dwarf has really been affected by Galactic tides.

\subsubsection{Sculptor}

Sculptor is also a difficult case. Its projected density profile is well described by a Plummer law, as would be expected for a relaxed system that has undergone tidal stripping, but the pericenter of its orbit seems too large for tides to have played a role. Indeed, we find that the tidal radius lies well beyond the stellar radius (assuming the orbital pericenter to be $r_{\text {peri }} \sim 76 \mathrm{kpc}$, see Table 2). Eq. 5 predicts a break at $\simeq 1 \mathrm{kpc}$ (indicated by the solid arrow in Fig. 5), but there is no obvious feature in the profile at that radius. We conclude that the "break" claimed by (Westfall et al. 2006) at $\approx 1.5 \mathrm{kpc}$ is $n o t$ tidal in origin but likely a result of fitting a King model to a profile that is best described by a power-law at large radii.

\subsubsection{Carina}

The presence of a break in the outer profile is probably clearest in the case of the Carina dSph (Majewski et al. 2005). As shown in Fig. 5, the surface density profile of this galaxy can be accurately fitted by a King model with concentration $c_{K}=4$ inside $R_{b}=0.70 \mathrm{kpc} \approx 4 R_{c}$. 


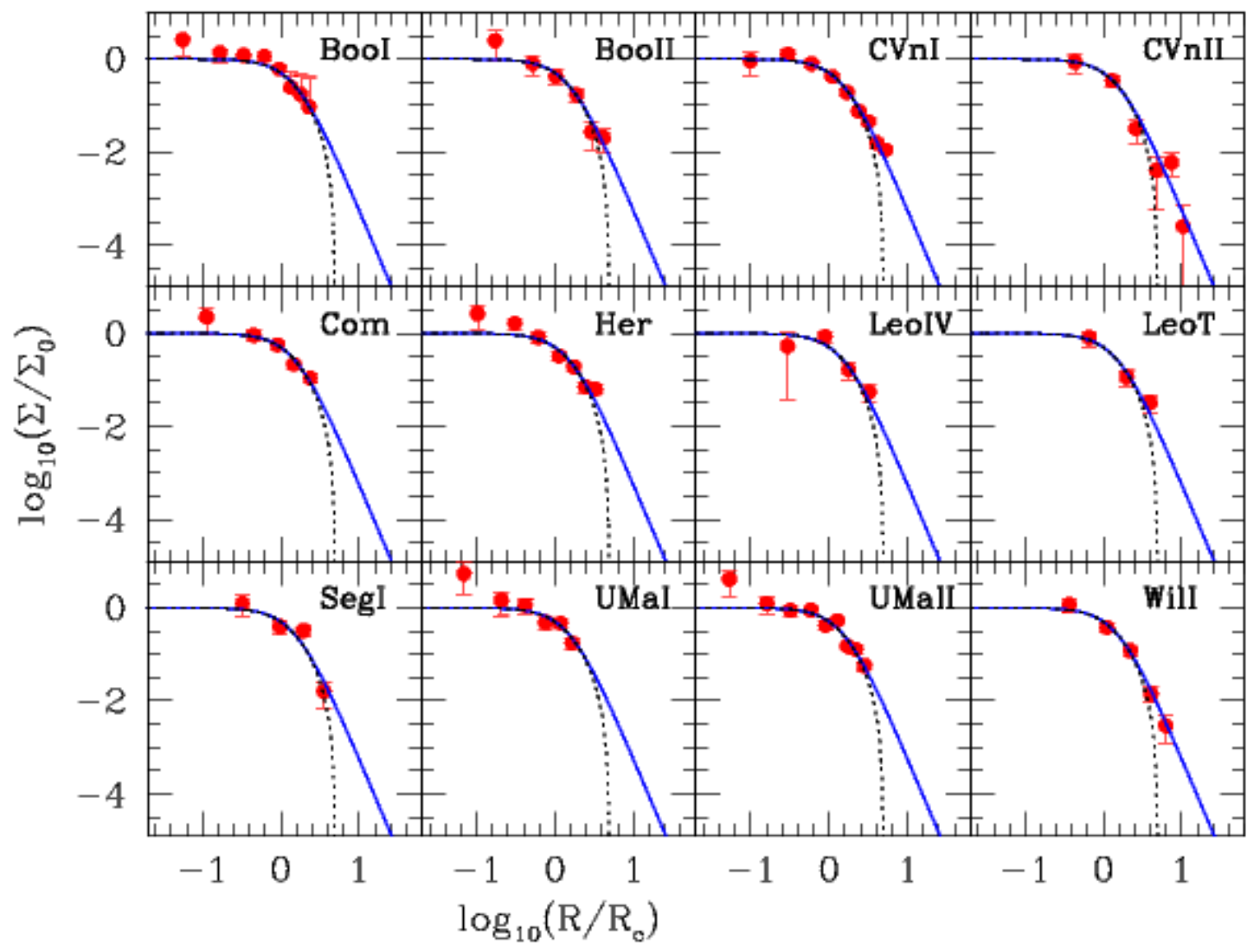

Fig. 6. - As Fig. 5 but for 12 of the recently-discovered, ultra-faint dSphs, as given by Martin et al. (2008). Each profile has been scaled to the central surface brightness and the core radius. Solid and dotted lines show the Plummer and King $\left(c_{K}=4\right)$ projected density profiles.

At that radius, the light profile shows a clear inflection point and the profile becomes much shallower out to the outermost radius surveyed. Muñoz et al. (2007) argue that this feature in the outer profile is tidal in origin.

Our results seem to disagree with this conclusion. According to our orbit estimate, the tidal radius of Carina at perigalacticon is roughly $\sim 4 R_{c}$, which means that Galactic tides could, in principle, have been important in this galaxy. However, eq. 5 indicates that the break should occur a little more than twice farther from the center than observed (see solid arrow in the Carina panel of Fig. 5). This would seem to suggest that the feature observed in Carina is not tidal in origin.

This conclusion is subject to a few caveats. Our analysis neglects projection effects that may result in the superposition of unbound stars onto the main core of the dwarf, as would be the case if we were seeing a tidal tail "end on". This may shift the break radius inwards, although by how much is not clear at this point. Also, it should be noted that measuring proper motions for stars at such distance $(\simeq 100 \mathrm{kpc})$ is an extremely difficult task. The possibility remains, therefore, that the orbit we assume for Carina (see Table 2 may be in error. Should a revised orbit in the future lead to better agreement between the predictions of eq. 5 and the observed location of the break, it would certainly lend support to the idea that this feature is tidal in origin and that Carina is in the process of shedding many of its stars into the Galactic halo.

\subsubsection{Ursa Minor}

The orbit of the Ursa Minor dSph implies a tidal radius well outside the luminous radius of the dwarf, casting doubt on a tidal interpretation for the "break" at $\sim 0.65$ kpc $\sim 3 R_{c}$ claimed by Martínez-Delgado et al. (2001) and Palma et al. (2003). This conclusion is supported by the fact that the predicted location of the tidal break, according to eq. 5. would be at roughly $\sim 7 \mathrm{kpc}$, or $\sim 20 R_{c}$, well beyond the region surveyed by available data. Unless the orbit for Ursa Minor is substantially in error, we conclude that the features in the outer profile of this dwarf are not tidal in origin.

\subsubsection{Sextans}

As in the case of the UMi dSph, the orbit of Sextans implies a tidal radius that falls beyond the region occupied by stars, which suggests that stellar stripping have not affected this galaxy. Interestingly, the irregular, patchy shape of Sextans (Irwin \& Hatzidimitriou 1995) has been commonly explained as a possible signature of tidal distortions. However, the proper motions recently estimated by Walker et al. (2008) place the orbital pericentre of Sextans at $\approx 70 \mathrm{kpc}$ and therefore seem to contradict this scenario. On the other hand, the density profile of Sextans is only known out to $\sim 4 R_{c}$ from the galaxy center, which proves insufficient to determine 
whether or not the luminous profile is truncated at large radii.

\subsection{Dwarfs with unknown orbits}

Of all the systems shown in Fig. 5 . Leo II and Draco do not have published proper motions, so it is not possible to estimate robustly the time elapsed since perigalacticon. Furthermore, the radial extent of the data available for Sextans $\left(R<3 R_{c}\right)$ is too small to allow for conclusive analysis. Leo II appears to be sharply truncated in the outer regions, but again the radial extent of the data is probably not good enough to warrant a more detailed assessment. Draco, on the other hand, has an outer profile that resembles a Plummer law, so it is possible in principle that tides may have played a role in the evolution of this system. A determination of the orbit of these systems, together with data that extend the profile to larger radii, are needed in order to assess the role of tides in their evolution.

\subsubsection{Ultra-faint dwarfs}

Fig. 6 shows the surface density profiles of 12 ultrafaint dwarfs recently discovered in SDSS data, as computed by Martin et al. (2008). The scaling in each profile is the same as in Fig. 5. Available data for the ultrafaint dwarfs clearly do not extend sufficiently far in order to allow for the same kind of analysis we carried out in the previous subsections for their brighter counterparts. Nevertheless, it is interesting that those galaxies with deep photometric data, e.g. CVn II and Willman I, seem to follow a Plummer profile in the outer regions. Although this is not a sufficient condition to guarantee that these systems have been strongly perturbed by tides, it implies that the possibility that at least some of these systems may have been shaped by tides should not be dismissed out of hand.

\section{SUMMARY}

We have used N-body simulations to identify signatures of ongoing or past tidal stripping that may be used to guide the interpretation of the observed surface density profile of dwarf spheroidals. Our models assume cosmologically motivated initial conditions, where the stellar component of a dSph is represented by a King sphere embedded within an NFW halo. The modeling assumes that dSphs are on eccentric orbits, so that tides operate over short periods of time, when the dwarf is at its orbital perigalacticon.

Our main findings may be summarized as follows.

- Only systems in orbits where the tidal radius (eq. 3) (measured at perigalacticon) is comparable to or smaller than the luminous radius of the dwarf are significantly affected by tides.

- The luminous profile of dwarfs that have undergone at least mild tidal mass loss is modified substan- tially, and approaches a Plummer law as the system gradually resettles into equilibrium after each pericentric passage.

- As the system re-equilibrates, the outer profile shows transient "bumps" or "breaks" at radii where the local crossing time exceeds the time elapsed since perigalacticon. For systems with known orbits, this provides a way of assessing whether breaks in the observed $\mathrm{dSph}$ profiles are actually tidal in origin.

Applied to the Sagittarius dwarf (where tidal stripping is beyond doubt), these results identify two features in the surface brightness profile that may be traced to its two last pericentric passages. Encouraged by this, we have also checked the plausibility of a tidal origin for the "breaks" reported in other dSphs.

We are able to rule out a possible tidal origin for the break reported in Leo I. Indeed, our results predict that any tidal break would occur at radii beyond those surveyed by current data. For Carina, our model indicates that the tidal break should occur at a radius twice as far as observed. We conclude that either the outer excess of stars in Carina is not tidal in origin or that the published proper motions for this system are in error.

Sculptor is an intriguing case; its profile matches well a Plummer law (suggesting that past tidal mass loss could have occurred), but the tidal radius seems too small for tides to have been important in this system. A similar comment applies to Draco, although there is no published orbit for this system, which hinders further analysis. Available data for Ursa Minor and Sextans do not probe the density profile far enough out to allow for meaningful constraints. We note, however, that the tidal radius for both systems are too large for tides to have been important during their evolution.

Published profiles for other Milky Way dSph companions do not probe sufficiently far to allow for conclusive assessment. Photometric surveys that extend surface brightness profiles beyond $\sim 10$ core radii, together with improved constraints on the orbital parameters of dSphs, are needed in order to establish whether Galactic tides actually play an active role in shaping the structure of our faintest and closest extragalactic neighbours.

JP acknowledges useful comments from M. Wilkinson, M. Bellazzini and the anonimous referee. JP and JFN would like to acknowledge the hospitality of the KITP at the University of California, Santa Barbara where part of this work was completed. This research was supported in part by the National Science Foundation under Grant No. NSF PHY05-51164. AWM acknowledges support from a Research Fellowship from the Royal Commission for the Exhibition of 1851 during much of this work. He also thanks Sara Ellison for additional financial assistance during this time.

\section{REFERENCES}

Aguerri, J. A. L., \& González-García, A. C. 2009, A\&A, 494, 891

Aguilar, L. A., \& White, S. D. M. 1986, ApJ, 307, 97

Battaglia, G., et al. 2006, A\&A, 459, 423

Bellazzini, M., Gennari, N., Ferraro, F. R., \& Sollima, A. 2004, MNRAS, 354,708
Bellazzini, M., et al. 2008, AJ, 136, 1147

Belokurov, V., et al. 2006a, ApJ, 647, L111

Belokurov, V., et al. 2006b, ApJ, 642, L137

Belokurov, V., et al. 2007, ApJ, 654, 897

Belokurov, V., et al. 2008, ArXiv e-prints, 807, arXiv:0807.2831 
Benson, A. J., Lacey, C. G., Baugh, C. M., Cole, S., \& Frenk, C. S. 2002, MNRAS, 333, 156

Bullock, J. S., Wechsler, R. H., \& Somerville, R. S. 2002, MNRAS, 329, 246

Caputo, F., Cassisi, S., Castellani, M., Marconi, G., \& Santolamazza, P. 1999, AJ, 117, 2199

Cole, S., Aragon-Salamanca, A., Frenk, C. S., Navarro, J. F., \& Zepf, S. E. 1994, MNRAS, 271, 781

Coleman, M. G., Jordi, K., Rix, H.-W., Grebel, E. K., \& Koch, A. 2007, AJ, 134, 1938

Diemand, J., Kuhlen, M., Madau, P., Zemp, M., Moore, B., Potter, D., \& Stadel, J. 2008, Nature, 454, 735

Dinescu, D. I., Girard, T. M., van Altena, W. F., \& López, C. E. 2005, ApJ, 618, L25

Fellhauer, M., Kroupa, P., Baumgardt, H., Bien, R., Boily, C. M., Spurzem, R., \& Wassmer, N. 2000, New Astronomy, 5, 305

Gilmore, G., Wilkinson, M. I., Wyse, R. F. G., Kleyna, J. T., Koch, A., Evans, N. W., \& Grebel, E. K. 2007, ApJ, 663, 948

González-García, A. C., Aguerri, J. A. L., \& Balcells, M. 2005, A\&A, 444, 803

Held, E. V., Clementini, G., Rizzi, L., Momany, Y., Saviane, I., \& Di Fabrizio, L. 2001, ApJ, 562, L39

Holmberg, E. 1950, Meddelanden fran Lunds Astronomiska Observatorium Serie II, 128, 1

Hubble, E. 1936, Contributions from the Mount Wilson Observatory / Carnegie Institution of Washington, 548, 1

Ibata, R., Martin, N. F., Irwin, M., Chapman, S., Ferguson, A. M. N., Lewis, G. F., \& McConnachie, A. W. 2007, ApJ, 671, 1591

Irwin M., Hatzidimitriou D., 1995, MNRAS, 277, 1354

Irwin, M. J., et al. 2007, ApJ, 656, L13

Irwin, M. J., Ferguson, A. M. N., Huxor, A. P., Tanvir, N. R., Ibata, R. A., \& Lewis, G. F. 2008, ApJ, 676, L17

Jaffe, W. 1987, Structure and Dynamics of Elliptical Galaxies, 127,511

Johnston, K. V., Choi, P. I., \& Guhathakurta, P. 2002, AJ, 124, 127

Kauffmann, G., White, S. D. M., \& Guiderdoni, B. 1993, MNRAS, 264, 201

Kazantzidis S., Magorrian J., Moore B., 2004, ApJ, 601, 37

Kazantzidis S., Zenter A., Kravtsov A., 2006, ApJ, 641, 647

King, I. 1962, AJ, 67, 471

King, I. R. 1966, AJ, 71, 64

Klimentowski, J., Łokas, E. L., Kazantzidis, S., Prada, F., Mayer, L., \& Mamon, G. A. 2007, MNRAS, 378, 353

Klypin, A., Kravtsov, A. V., Valenzuela, O., \& Prada, F. 1999, ApJ, 522, 82

Klypin, A., Zhao, H., \& Somerville, R. S. 2002, ApJ, 573, 597

Koch, A., Wilkinson, M. I., Kleyna, J. T., Gilmore, G. F., Grebel, E. K., Mackey, A. D., Evans, N. W., \& Wyse, R. F. G. 2007, ApJ, 657, 241

Łokas, E. L., Klimentowski, J., Kazantzidis, S., \& Mayer, L. 2008, MNRAS, 390, 625

Madau, P., Diemand, J., \& Kuhlen, M. 2008, ApJ, 679, 1260

Majewski, S. R., Ostheimer, J. C., Patterson, R. J., Kunkel, W. E., Johnston, K. V., \& Geisler, D. 2000, AJ, 119, 760

Majewski, S. R., Skrutskie, M. F., Weinberg, M. D., \& Ostheimer, J. C. 2003, ApJ, 599, 1082

Majewski, S. R., et al. 2005, AJ, 130, 2677

Majewski, S. R., et al. 2007, ApJ, 670, L9

Martin, N. F., Ibata, R. A., Irwin, M. J., Chapman, S., Lewis, G. F., Ferguson, A. M. N., Tanvir, N., \& McConnachie, A. W. 2006, MNRAS, 371, 1983

Martin, N. F., Ibata, R. A., Chapman, S. C., Irwin, M., \& Lewis, G. F. 2007, MNRAS, 380, 281

Martin, N. F., de Jong, J. T. A., \& Rix, H.-W. 2008, ApJ, 684, 1075

Martínez-Delgado, D., Alonso-García, J., Aparicio, A., \& Gómez-Flechoso, M. A. 2001, ApJ, 549, L63

Mastropietro, C., Moore, B., Mayer, L., Debattista, V. P., Piffaretti, R., \& Stadel, J. 2005, MNRAS, 364, 607

Mateo M.L., 1998, ARA\&A, 36, 435
Mateo, M., Olszewski, E. W., \& Walker, M. G. 2008, ApJ, 675, 201

Mayer, L., Moore, B., Quinn, T., Governato, F., \& Stadel, J. 2002, MNRAS, 336, 119

McConnachie, A. W., Peñarrubia, J., \& Navarro, J. F. 2007, MNRAS, 380, L75

McConnachie, A., et al. 2008, arXiv:0806.3988

Moore, B., Ghigna, S., Governato, F., Lake, G., Quinn, T., Stadel, J., \& Tozzi, P. 1999, ApJ, 524, L19

Muñoz, R. R., et al. 2005, ApJ, 631, L137

Muñoz, R. R., et al. 2006, ApJ, 649, 201

Muñoz, R. R., Majewski, S. R., \& Johnston, K. V. 2007, ArXiv e-prints, 712, arXiv:0712.4312

Muñoz, R. R., Majewski, S. R., \& Johnston, K. V. 2008, ApJ, 679,346

Navarro, J. F. 1990, MNRAS, 242, 311

Navarro, J. F., Frenk, C. S., \& White, S. D. M. 1996, ApJ, 462, 563

Navarro J., Frenk C.S., White S.D.M., 1997, ApJ, 490, 493 (NFW)

Oh, K. S., Lin, D. N. C., \& Aarseth, S. J. 1995, ApJ, 442, 142

Palma, C., Majewski, S. R., Siegel, M. H., Patterson, R. J., Ostheimer, J. C., \& Link, R. 2003, AJ, 125, 1352

Peñarrubia, J., \& Benson, A. J. 2005, MNRAS, 364, 977

Peñarrubia, J., McConnachie, A. W., \& Navarro, J. F. 2008, ApJ, 672, 904 (Paper I)

Peñarrubia, J., Navarro, J. F., \& McConnachie, A. W. 2008, ApJ, 673, 226 (Paper II)

Piatek, S., Pryor, C., Olszewski, E. W., Harris, H. C., Mateo, M. Minniti, D., \& Tinney, C. G. 2003, AJ, 126, 2346

Piatek, S., Pryor, C., Bristow, P., Olszewski, E. W., Harris, H. C., Mateo, M., Minniti, D., \& Tinney, C. G. 2005, AJ, 130, 95

Piatek, S., Pryor, C., Bristow, P., Olszewski, E. W., Harris, H. C., Mateo, M., Minniti, D., \& Tinney, C. G. 2006, AJ, 131, 1445

Piatek, S., Pryor, C., Bristow, P., Olszewski, E. W., Harris, H. C., Mateo, M., Minniti, D., \& Tinney, C. G. 2007, AJ, 133, 818

Plummer, H. C. 1911, MNRAS, 71, 460

Power, C., Navarro, J. F., Jenkins, A., Frenk, C. S., White, S. D. M., Springel, V., Stadel, J., \& Quinn, T. 2003, MNRAS, 338,14

Read, J. I., Wilkinson, M. I., Evans, N. W., Gilmore, G., \& Kleyna, J. T. 2006, MNRAS, 367, 387

Rizzi, L., Held, E. V., Saviane, I., Tully, R. B., \& Gullieuszik, M. 2007, MNRAS, 380, 1255

Simon, J. D., \& Geha, M. 2007, ApJ, 670, 313

Sohn, S. T., et al. 2007, ApJ, 663, 960

Springel, V., et al. 2008, arXiv:0809.0898

Stoehr, F., White, S. D. M., Tormen, G., \& Springel, V. 2002, MNRAS, 335, L84

Strigari, L. E., Bullock, J. S., Kaplinghat, M., Simon, J. D., Geha, M., Willman, B., \& Walker, M. G. 2008, Nature, 454, 1096

Walcher, C. J., Fried, J. W., Burkert, A., \& Klessen, R. S. 2003, A\&A, 406, 847

Walker, M. G., Mateo, M., Olszewski, E. W., Gnedin, O. Y., Wang, X., Sen, B., \& Woodroofe, M. 2007, ApJ, 667, L53

Walker, M. G., Mateo, M., \& Olszewski, E. W. 2008, ApJ, 688, L75

Westfall, K. B., Majewski, S. R., Ostheimer, J. C., Frinchaboy, P. M., Kunkel, W. E., Patterson, R. J., \& Link, R. 2006, AJ, 131,375

White, S. D. M. 1987, Structure and Dynamics of Elliptical Galaxies, 127, 339

White, S. D. M., \& Rees, M. J. 1978, MNRAS, 183, 341

Willman, B., et al. 2005, ApJ, 626, L85

Zucker, D. B., et al. 2004, ApJ, 612, L121

Zucker, D. B., et al. 2006, ArXiv Astrophysics e-prints, arXiv:astro-ph/0601599

Zucker, D. B., et al. 2006, ApJ, 643, L103

Zucker, D. B., et al. 2006, ApJ, 650, L41

Zucker, D. B., et al. 2007, ApJ, 659, L21 
TABLE 1

ORBITAL PARAMETERS OF OUR MODELS

\begin{tabular}{|c|c|c|c|c|c|c|c|}
\hline$r_{\text {peri }}: r_{\text {apo }}$ & $\begin{array}{l}r_{\text {peri }} \\
{[\mathrm{kpc}]}\end{array}$ & $\begin{array}{c}T_{r} \\
{[\mathrm{Gyr}]}\end{array}$ & $\begin{array}{l}r_{\text {tid }} \\
{\left[r_{s}^{d}\right]}\end{array}$ & $\begin{array}{c}M^{d}\left(<r_{\mathrm{tid}}\right)^{\top} \\
{\left[M_{\mathrm{tot}}^{d}\right]}\end{array}$ & $f_{\text {bound }}^{d}{ }^{\dagger}$ & $\begin{array}{l}f_{\text {bound }}^{\star}{ }^{\ddagger} \\
\left(c_{k}=5\right)\end{array}$ & $\begin{array}{l}f_{\text {bound }}^{\star} \\
\left(c_{k}=10\right)\end{array}$ \\
\hline $1: 50$ & 3.6 & 2.94 & 0.083 & $1.35 \times 10^{-3}$ & $6.6 \times 10^{-2}$ & 0.91 & 0.50 \\
\hline 1:100 & 1.8 & 2.80 & 0.036 & $2.75 \times 10^{-4}$ & $3.7 \times 10^{-2}$ & 0.65 & 0.30 \\
\hline $1: 200$ & 0.9 & 2.22 & 0.017 & $6.50 \times 10^{-5}$ & $2.0 \times 10^{-2}$ & 0.38 & 0.17 \\
\hline
\end{tabular}

I estimated for an unperturbed model at the pericentric distance

${ }^{\dagger}$ calculated at the apocentre following the first pericentric passage

$$
{ }^{\ddagger} \text { Segregation } R_{k} / r_{\text {max }}^{d}=0.1
$$

TABLE 2

Orbital Parameters of Local Group Satellites with Known orbit

\begin{tabular}{lcccccccc}
\hline \hline Galaxy & Sgr (last) & Sgr (penultim.) & Leo I & Sculptor & Carina & Ursa Minor & Fornax & Sextans \\
\hline$D(\mathrm{kpc})$ & $25 \pm 2$ & $25 \pm 2$ & $250 \pm 30$ & $87 \pm 4$ & $100 \pm 5$ & $76 \pm 3$ & $138 \pm 8$ & $86 \pm 4$ \\
$l\left(^{\circ}\right)$ & 6.7 & 6.7 & 226 & 283 & 260 & 105 & 237 & 243 \\
$b\left(^{\circ}\right)$ & -24.4 & -24.4 & +49.0 & -84.5 & -22.2 & +44.8 & -65.8 & +43 \\
$v_{r, h}(\mathrm{~km} / \mathrm{s})$ & +137 & +137 & +259 & +110 & +223 & -247 & +53 \\
$\mu_{l}(\mathrm{mas} /$ year $)$ & $-2.58 \pm 0.20$ & $-2.58 \pm 0.20$ & - & $-0.09 \pm 0.14$ & $-0.08 \pm 0.09$ & $0.51 \pm 0.17$ & $0.29 \pm 0.05$ & $0.24 \pm 0.42$ \\
$\mu_{b}$ (mas/year) & $2.07 \pm 0.20$ & $2.07 \pm 0.20$ & - & $0.01 \pm 0.13$ & $0.25 \pm 0.09$ & $0.20 \pm 0.17$ & $0.52 \pm 0.05$ & $-0.14 \pm 0.42$ \\
$R_{c}(\mathrm{kpc})$ & 1.50 & 1.50 & 0.17 & 0.1 & 0.18 & 0.20 & 0.40 & 0.32 \\
$\sigma_{0}(\mathrm{~km} / \mathrm{s})$ & $9.6 \pm 1.0$ & $9.6 \pm 1.0$ & $8.8 \pm 1.3$ & $6.6 \pm 1.0$ & $6.8 \pm 1.0$ & $9.3 \pm 2.0$ & $11.1 \pm 0.7$ & $6.6 \pm 0.7$ \\
$r_{\text {peri }}(\mathrm{kpc})$ & $16.5 \pm 2.4$ & $16.5 \pm 2.4$ & 0 & $76 \pm 4$ & $24 \pm 5$ & $48 \pm 8$ & $129 \pm 17$ & $69 \pm 9$ \\
$t-t_{p}(\mathrm{Gyr})$ & $0.023 \pm 0.011$ & $1.0 \pm 0.2$ & $1.02 \pm 0.12$ & $0.28 \pm 0.03$ & $0.81 \pm 0.12$ & $1.5 \pm 0.3$ & $6.3 \pm 2.9$ & $0.32 \pm 0.08$ \\
$R_{b, \text { obs }}(\mathrm{kpc})$ & $7.3 \pm 0.6$ & $7.3 \pm 0.6$ & $0.58 \pm 0.14$ & $1.3 \pm 0.2$ & $0.70 \pm 0.14$ & $0.65 \pm 0.10$ & $2.4 \pm 0.2$ & - \\
$R_{b, \text { pred }}(\mathrm{kpc})$ & $0.14 \pm 0.08$ & $5.4 \pm 2$. & $5.1 \pm 1.3$ & $1.04 \pm 0.11$ & $3.1 \pm 0.4$ & $7.9 \pm 1.5$ & $39 \pm 18$ & $1.2 \pm 0.4$ \\
refs. & $1,2,3$ & $1,2,3$ & $4,5,6$ & $7,8,9$ & $5,10,11,12$ & $5,13,14,15$ & $5,16,17$ & 5,18 \\
\hline
\end{tabular}

References. - (1) Majewski et al. (2003); (2) Dinescu et al. (2005); (3) Bellazzini et al. (2008); (4) Caputo et al. (1999); (5) Mateo (1998, 2008); (6) Koch et al. (2007); (7) Walcher et al. (2003); (8) Westfall et al. (2006); (9) Piatek et al. (2006); (10) Majewski et al. (2000, 2005); (11) Muñoz et al. (2006); (12) Piatek et al. (2003); (13) Palma et al. (2003); (14) Muñoz et al. (2005); (15) Piatek et al. (2005); (16) Rizzi et al. (2007); (17) Piatek et al. (2007); (18) Walker et al. (2008) 